\title{
GUEST REVIEWERS FOR THE JOURNAL OF THORACIC AND CARDIOVASCULAR SURGERY
}

The Editor and members of the Advisory Editorial Board wish to express their appreciation to the following Guest Reviewers for the time and valuable assistance given during the past year in the review of manuscripts for this Journal.

Michael A. Acker

Cary W. Akins

Mario Albertucci

Gabriel S. Aldea

Bradley S. Allen

Mark S. Allen

Nasser K. Altorki

James M. Anderson

Harry L. Anderson III

Kit V. Arom

Renato Assad

Safuh Attar

Leon Axel

Carl L. Bacher

John C. Baldwin

Hendrick B. Barner

Robert H. Beekman

Willıam Robert Bell

John R. Benfield

Lee N. Benson

Alvise Bernabeı

Friedhelm Beyersdorf

John D. Birkmeyer

Chrss I. Blauth

Willy D. Boeckx

Daniel K. Bogen

Steven F. Bolling

Harvey S. Borovetz

Hans G. Borst

Michael Bousamra II

Edward L. Bove

Scott M. Bradley

Nancy Bridges

Richard F. Brodman

James M. Brown

W Morrss Brown III

Redmond Burhe

Daniel Burkhoft

John Butler

Frederick W. Campbell

Larry R. Casey

Jacques C. Chachques

Sylvain Chauvaud

Aurelıo Chaux
W. Randolph Chitwood George T. Christakis

Jan T. Christenson

Taylor Chung

Richard P. Cochran

Michael V. Cohen

Lawrence H. Cohn

Patricia L. Cole

Robert W. Colman

Joel D. Cooper

Joseph S. Coselli

Delos M. Cosgrove

Joseph M. Craver

Jochen Cremer

Stephen E. Cyran

Willaard M. Daggett

Richard S. D'agostıno

Pat O. Daily

Thomas M Daniel

Gordon K. Danielson

Tirone E. David

Willıam R. Davidson, Jr

Victor G. Davila-Roman

R. Duane Davis

Marc R. de Leval

Malcolm DeCamp

Pedro J. del Nido

Ralph E. Delius

Walter P. Dembitsky

Claude Deschamps

George J. Despotis

Marc L Dickstein

Verdı J DiSesa

Donald B. Doty

Steve Downing

Carolyn M. Dresler

Davis C. Drunkwater, Jr

Carlos M Gomez Duran

Stanley W. Dzıuban

Robert C. Eberhart

Thomas M. Egan

John Alex Elefteriades

Ronald C. Elkins

Richard M. Engelman
Volkmar Falk

Richard Feins

Stanley C. Fell

Bruce Ferguson

Mark K. Ferguson

Victor A. Ferrans

Aaron B. Fisher

Carol A Fisher

Willem Flameng

Oscar Howard Frazıer

Satoshı Furukawa

Henning A. Gatssert

J. Willam Gaynor

Elliot T. Gelfand

Cynthia Gemmell

David T. George

A. Marc Gillınov

Robert J. Ginsberg

Donald D. Glower, Jr

Robert C. Gorman

Joseph H. Gorman III

John P. Gott

Glenn P. Gravlee

Randall B. Griepp

Bartley P. Griftith

Hermes C. Grillo

Fredenck L. Grover

Gary Grunkemeter

Steven Guyton

C Erih Hach

Jeffrey A. Hagen

Per-Otto Hagen

J. Alex Haller

John W Hammon. Jr

Graeme L. Hammond

Edward L. Hannan

David H. Harpole. Jr

Frank E Harrell

Axel Haverich

Stephen R. Hazelrigg

Guo-We1 He

Eric J. Heyer

Bradley J. Hindman

Loren F. Hiratzka
Bradley O. Hofer

Willaam L. Holman

E. Carmack Holmes

Richard A. Hopkins

Jan Horrow

Keith A. Horvath

Renee S. Houtz

Charles B Huddleston

Tony E. Hugli

Yasuharu Imai

Marshall L. Jacobs

Stuart W. Jamieson

W. R. Erıc Jamieson

Erik W. L. Jansen

Valluvan Jeevanandam

Michael E. Jessen

David R. Jobes

Robert G. Johnson

David W. Johnstone

Kent W. Jones

Robert H. Jones

William D. Jordan

Robert B. Karp

Teruhısa Kazui

John A. Kern

Shafique Keshavjee

Steve Khan

Shukrı F Khurı

Paul Kurschner

Leslie J. Kohman

Rohert L Kormos

Mark J. Krasna

Jacqueline Kreutzer

Irving L. Kron

Irvin B. Krukenkamp

Rodney J. Landreneau

Edward B. Lankford

Albert C. Lardo

Michael Lauer

Gerald M Lawrie

Harold L. Lazar

Jean-Louss LeClerc

Jerrold H. Levy

Robert Levy 


\author{
Alex G. Little \\ James E. Lock \\ Yves A. G. Louagie \\ Robert Bruce Love \\ James E. Lowe \\ Flavian M. Lupinetti \\ Michael J. Mack \\ Michael Maddaus \\ Joren C. Madsen \\ George J. Magovern, Jr \\ John D. Mannion \\ Daniel B. Mark \\ Nael Martini \\ Douglas J. Mathisen \\ Constantine Mavroudis \\ John E. Mayer \\ Patrick M. McCarthy \\ Brıan W. McCrindle \\ Andrew D. McCulloch \\ David C. McGiffin \\ Edwin C. McGough \\ Lynn B. McGrath \\ Roger B. B. Mee \\ Philippe Menasché \\ Eric N. Mendeloff \\ Scot H. Merrick \\ Dan M. Meyer \\ Noel L. Mills \\ R. Scott Mitchell \\ Adrian Moran \\ Clifton F. Mountain \\ Derek D. Muehrcke \\ John M. Murkin \\ David C. Naftel \\ Navin C. Nanda \\ Keith Naunheim \\ C. A. Nienaber \\ Stefan Niewiarowski \\ Gregory A. Nuttall \\ Celia M. Oakley
}

Mark F. O’Brien

Gordon N. Olınger

Mark Orrınger

Richard A. Ott

Eivind Ovrum

Mehmet C. Oz

Walter E. Pae, Jr

Peter C. Paurolero

Victor Parsonnet

Michael D. Pasque

Harvey I. Pass

F. Griffith Pearson

Carlos Pellegrini

William Piccione, Jr

Richard N. Pierson III

Jeffrey L. Platt

Timothy Pohlman

Karin Przyklenk

Wiltred B. Pugsley

Joe B. Putnam, Jr

A. Koneti Rao

Vivek Rao

Mark B. Ratcliffe

Federico Rea

V. Mohan Reddy

Mark Redmond

Carolyn E. Reed

Keith Reemtsma

Bruce A. Reitz

Herbert Y. Reynolds

Thomas W. Rice

Wayne E. Richenbacher

Christine S. Rinder

Henry M. Rinder

Gary W. Roach

Robert C. Robbins

Chrıs K. Rokkas

Bruce Rosengard

Todd K. Rosengart

Tomas A. Salerno
Joseph W. Sassani

Hartzell V. Schaff

John J. Schier

Frederick J. Schoen

David Schrump

Walter J. Scott

Michael Sefton

Frank W. Sellke

Alan Serraf

Amrik Shah

Robert Shamberger

Barry Siegel

Norman A. Silverman

Colleen F. Sintek

Craig R. Smith

W. Roy Smythe

Seng-Jaw Soong

Francis G. Spinale

Vaughn A. Starnes

Mark M. Stecker

Larry W. Stephenson

David M. Stern

Valavanur A. Subramanian

Benjamın Sun

Sudhir Sundaresan

Thoralf M. Sundt

Lars Svensson

Julie A. Swain

Scott Swanson

Kolchi Tabayashi

David P. Taggart

Stanley K. C. Tam

John Tarbell

Kenneth M Taylor

Henk te Velthuis

Alfred J. Tector

Gaetano Thiene

Thomas R. J. Todd

Victor F. Trastek

Thomas Treasure
John Triedman

Malcolm Turner

Daniel J. Ullyot

Ross M. Ungerleider

Harold Urschel. Jr

Richard Van Praagh

Peter Van Trigt

Robert C. Vannuces

Jean-Louis J. Vanoverschelde

Victoria L. Vetter

Vibeke Videm

Gus J. Vlahakes

Ludwig K. von Segesser

William R. Wagner

Alexander M. Walker

Edward P. Walsh

James Ware

Alan G Wasserstein

Paul F. Waters

Clinton R. Webb

Howard S. Weber

Karl T. Weber

Richard D. Weisel

Gil Wernovsky

David Wessel

Steven Westaby

Mark Wick

Samuel A. Wickline

Walter G. Wolfe

Douglas Wood

Cameron D. Wright

Jerome Yager

A. Charles Yankah

J. Nilas Young

Pat L. Yudkin

Warren M. Zapol

Joseph B. Zwischenberger 